\title{
Different regimes of low-frequency fluctuations in vertical-cavity surface-emitting lasers
}

\author{
Marc Sciamanna \\ Service d'Electromagnétisme et de Télécommunications, Faculté Polytechnique de Mons, 31 Boulevard Dolez, \\ B-7000 Mons, Belgium \\ Cristina Masoller \\ Instituto de Física, Facultad de Ciencias, Universidad de la República, Igua 4225, Montevideo 11400, Uruguay
}

Neal B. Abraham

DePauw University, Greencastle, Indiana 46135-0037

Fabien Rogister, Patrice Mégret, and Michel Blondel

Service d'Electromagnétisme et de Télécommunications, Faculté Polytechnique de Mons, 31 Boulevard Dolez, B-7000 Mons, Belgium

Received July 11, 2002; revised manuscript received August 6, 2002

\begin{abstract}
A numerical study of vertical-cavity surface-emitting lasers with optical feedback demonstrates the existence of two different types of low-frequency fluctuations (LFFs). The competition of two equally dominant polarization modes characterizes one type of LFF, while the other type is characterized by power drops in a dominant polarization mode and power bursts in the orthogonal depressed mode. We characterize and compare these two types of LFFs on the basis of their polarization properties and their dependency on the laser parameters. We show furthermore that a transition is possible from one type of LFF to the other, depending on the values of the linear anisotropies of the vertical-cavity surface-emitting laser cavity. () 2003 Optical Society of America
\end{abstract}

OCIS codes: $190.3100,250.7260$.

\section{INTRODUCTION}

The vertical-cavity surface-emitting laser (VCSEL) has received much attention since its proposal by K. Iga. ${ }^{1}$ The VCSEL shows up as a key device for optical telecommunication systems and all optical signal handling. It exhibits many advantages over the conventional edgeemitting semiconductor laser $(\mathrm{EEL})^{2}$ such as a singlelongitudinal-mode operation, low threshold current, circular beam, low cost, and a dense packing capability. VCSELs are also interesting from a fundamental viewpoint when considering their light polarization. The polarization of the emitted light is not fixed and often switches between two orthogonal, linearly polarized (LP) states $(x$ and $y) .^{3}$ This polarization switching is a drawback in polarization-sensitive applications but on the other hand may lead to new and interesting applications for optical signal processing. ${ }^{4}$

When using VCSELs in optical telecommunication systems, a small amount of light is inevitably fed back into the VCSEL cavity as the result, for example, of parasitic reflections at the cleaved end of a fiber or from the disk in CD players. As with any semiconductor laser, VCSELs are sensitive to optical feedback. It was first thought that VCSELs may be relatively immune to optical feedback thanks to their high output-mirror reflectivity, but this effect is offset by the short VCSEL intracavity round trip. As a result, the feedback rate, i.e., the fraction of power reflected back to the VCSEL per unit of time, is similar to that of EELs for a given value of external mirror reflectivity. First studies of VCSELs subject to optical feedback have identified five typical dynamic regimes that have previously been reported in EELs, such as linewidth narrowing or broadening, external-cavity mode hopping, stable laser output, chaotic dynamics, and laser restabilization. ${ }^{5}$ However, the polarization properties of VCSELs also lead to new and interesting phenomena when VCSEL are coupled to an external cavity. We can cite, for example, polarization-switching phenomena in polarized optical feedback, ${ }^{6,7}$ polarization self-modulation in VCSELs coupled to optical feedback through a quarter-wave plate, ${ }^{8-14}$ optical-feedbackinduced polarization mode hopping, and generalized multistability. ${ }^{15}$ New applications of VCSELs for optical telecommunications may be proposed based on these delay-induced dynamics. In particular, VCSELs with delayed feedback may generate vectorial chaos in which not only the field amplitude and phase are chaotic but also the polarization. Vectorial chaos may be useful for multichannel secure communication systems. ${ }^{16}$ On the other hand, high-frequency oscillations resulting from polarization self-modulation may be 
used for optical clock recovery in all optical regeneration systems or as a stable source of microwave oscillations. ${ }^{14}$

In this paper we study the feedback-induced, LFF regime. In this regime the laser intensity (observed on a nanosecond time scale) exhibits sudden random drops toward zero, recovering gradually to its original value (for a review see Ref. 17). We show that the coexistence of two polarization modes allows VCSELs to present two different types of LFFs that cannot be resolved by studying the total output intensity but that can be clearly distinguished by studying the polarization-resolved modal intensities. Type I is characterized by competition between the $x$ - and $y$-polarization modes with nearly equal power; type II is characterized by intensity drops in the dominant polarization mode and intensity bursts in the depressed mode. Both LFF types are found in the same model, but their presence depend on the laser and feedback parameters. We analyze the origin of the two LFF types and show a route to type I LFF involving elliptically polarized states in a bifurcation cascade. We also show that a transition from one type of LFF to the other might be observed by modifying the frequency difference and/or the gain-to-loss ratio of both polarization modes. Our results yield insight into polarization dynamics of delayed VCSELs and motivate new experiments with VCSELs coupled to long external cavities. We analyze an example of vectorial chaos that appears to be a drawback for photonic systems including VCSELs but that might be useful as a source for chaotic communication systems.

The plan of this paper is as follows. In Section 2 we present the model, which is an extension of the San Miguel-Feng-Moloney (SFM) model ${ }^{18}$ that includes external optical feedback by taking into account a single reflection in the external cavity. Section 3 presents the two types of LFF that occur in the model and that might be clearly distinguished on the basis of their polarization properties. Section 4 demonstrates that varying the birefringence and/or the gain-to-loss ratio of the polarization modes might induce a transition from one type of LFF to the other. Section 5 analyzes the fast pulsating dynamics underlying the two types of LFF, especially the phase coherence properties of the modal intensities. Section 6 shows that the two types of LFF are also found on an alternative model that couples two polarization modes with different frequencies and gains through nonlinear gain-saturation coefficients. ${ }^{19}$ Our conclusions are presented in Section 7.

\section{MODEL}

Our numerical simulations are based on the SFM equations ${ }^{18}$ extended to isotropic optical feedback as

$$
\begin{aligned}
\dot{E}_{x}= & \kappa(1+i \alpha)\left[(N-1) E_{x}+i n E_{y}\right] \\
& -\left(\gamma_{a}+i \gamma_{p}\right) E_{x}+f E_{x}(t-\tau) \exp \left(-i \phi_{f}\right) \\
& +\left[\beta_{\mathrm{sp}}(N+n) / 2\right]^{1 / 2} \xi_{1}+\left[\beta_{\mathrm{sp}}(N-n) / 2\right]^{1 / 2} \xi_{2},
\end{aligned}
$$

$$
\begin{aligned}
\dot{E}_{y}= & \kappa(1+i \alpha)\left[(N-1) E_{y}-i n E_{x}\right] \\
& +\left(\gamma_{a}+i \gamma_{p}\right) E_{y}+f E_{y}(t-\tau) \exp \left(-i \phi_{f}\right) \\
& -i\left[\beta_{\mathrm{sp}}(N+n) / 2\right]^{1 / 2} \xi_{1}+i\left[\beta_{\mathrm{sp}}(N-n) / 2\right]^{1 / 2} \xi_{2} \\
\dot{N}= & -\gamma_{N}\left[N-\mu+N\left(\left|E_{x}\right|^{2}+\left|E_{y}\right|^{2}\right)\right. \\
& \left.+i n\left(E_{y} E_{x}^{*}-E_{x} E_{y}^{*}\right)\right] \\
\dot{n}= & -\gamma_{s} n-\gamma_{N}\left[n\left(\left|E_{x}\right|^{2}+\left|E_{y}\right|^{2}\right)\right. \\
& \left.+i N\left(E_{y} E_{x}^{*}-E_{x} E_{y}^{*}\right)\right]
\end{aligned}
$$

where $E_{x}$ and $E_{y}$ are the slowly varying amplitudes of the linearly polarized components of the electric field, $N$ is the total carrier difference between conduction and valence bands, and $n$ is the difference between the population inversions of the spin-up and spin-down radiation channels. $\kappa$ is the field decay rate, $\gamma_{N}$ is the decay rate of $N, \gamma_{s}$ is the spin-flip relaxation rate, $\alpha$ is the linewidth enhancement factor, $\mu$ is the normalized injection current $(\mu=1$ at threshold), $\gamma_{a}$ is the linear dichroism, and $\gamma_{p}$ is the linear birefringence.

The terms proportional to $E_{x, y}(t-\tau)$ account for a delayed reflection from the external mirror (multiple reflections are neglected). $\quad \tau$ is the external cavity round-trip time, $f$ is the feedback rate, and $\phi_{f}=\omega_{0} \tau$ is the feedback phase, where $\omega_{0}$ is the optical frequency of the polarization mode in the absence of birefringence and feedback.

Spontaneous emission noise is taken into account through the last two terms in Eqs. (1) and (2). $\beta_{\mathrm{sp}}$ is the spontaneous emission rate and $\xi_{1}, \xi_{2}$ are two uncorrelated white noises with zero mean and unitary variance.

\section{TWO DIFFERENT TYPES OF LFF REGIMES}

In our simulations we consider the following parameters fixed ${ }^{20}: \quad \kappa=300 \mathrm{~ns}^{-1}, \quad \gamma_{N}=1 \mathrm{~ns}^{-1}, \quad \alpha=3$, and $\phi_{f}$ $=6 \mathrm{rad}$. Figures 1 and 2 display two different types of LFFs. Drops of the total intensity are observed in both cases [Figs. 1(a) and 2(a)], but the underlying polarization dynamics are very different. Type I is characterized by the competition of the $x$ - and $y$-polarization modes with nearly equal power [Figs. 1(b) and (c)], while type II is characterized by power drops in the dominant $y$-mode and power bursts in the depressed $x$-mode [Figs. 2(b) and (c)].

Type I LFF was previously found in simulations of the SFM model, ${ }^{20}$ while type II was observed experimentally and numerically in Ref. 21. It is worth noting that the model of Ref. 21 is also based on the SFM model but differs from Eqs. (1)-(4) in two respects. First Ref. 21 includes multiple reflections in the external cavity. The high reflectivity of the output mirror in VCSELs might justify inclusion of multiple external-cavity round trips even for a low feedback rate, as first suggested by Law and Agrawal. ${ }^{22}$ Inclusion of multiple round-trip terms in the field equation would most probably yield a better quantitative agreement with experiments, since it adds correction factors to the gain and frequency of the steadystate, external-cavity modes. ${ }^{23,24}$ However our results show that both types of LFF are complementary and co- 

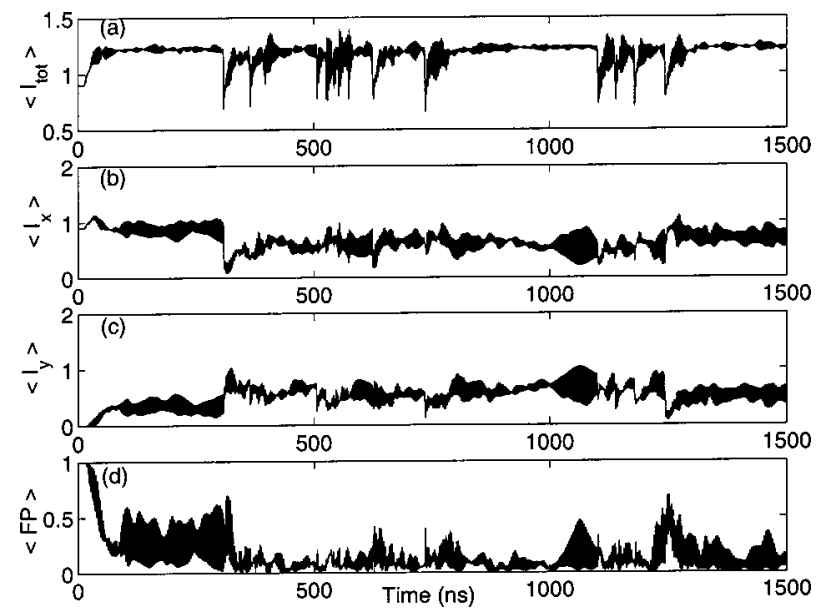

Fig. 1. Type I LFF. (a) The total intensity $I_{\text {tot }}$, (b), (c), the modal intensities $I_{x}$ and $I_{y}$, and (d) the degree of polarization $F P$ are plotted as a function of time after averaging on $1 \mathrm{~ns}$. The brackets indicate averaged variables. The parameters are $\gamma_{a}$ $=0.1 \mathrm{~ns}^{-1}, \gamma_{p}=10 \mathrm{~ns}^{-1}, \gamma_{s}=50 \mathrm{~ns}^{-1}, \mu=1.8, f=60 \mathrm{~ns}^{-1}$, $\beta_{\mathrm{sp}}=10^{-6} \mathrm{~ns}^{-1}, \tau=3 \mathrm{~ns}$.

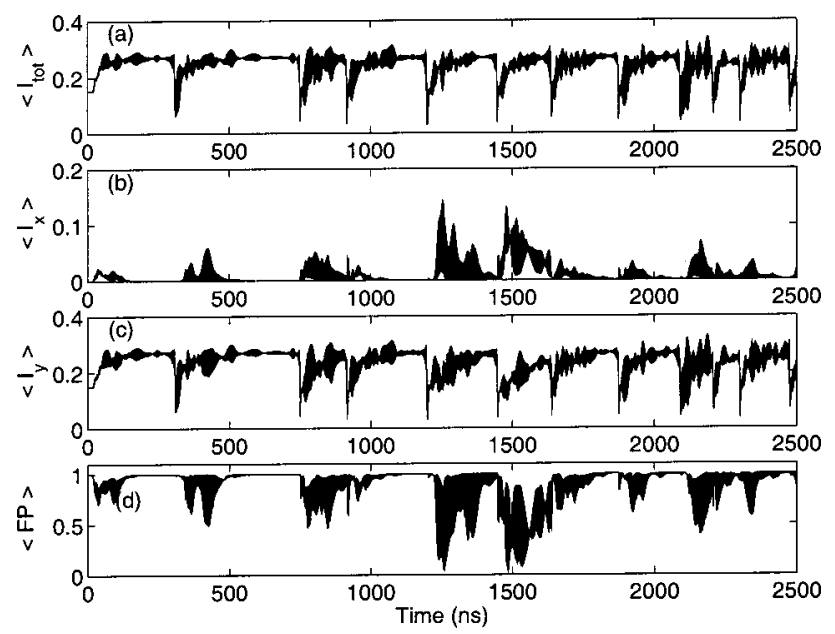

Fig. 2. Same as Fig. 1 but showing type II LFF. The parameters are $\gamma_{a}=0.5 \mathrm{~ns}^{-1}, \gamma_{p}=2 \mathrm{~ns}^{-1}, \gamma_{s}=50 \mathrm{~ns}^{-1}, \mu=1.1, f$ $=40 \mathrm{~ns}^{-1}, \beta_{\mathrm{sp}}=10^{-6} \mathrm{~ns}^{-1}, \tau=3 \mathrm{~ns}$.

exist in the same model [Eqs. (1)-(4)] using a single round-trip term in the external cavity as in the LangKobayashi approximation. ${ }^{25}$ As a first conclusion, multiple feedback-delay terms are not responsible for the type II LFF reported experimentally in Ref. 21. As we demonstrate below in Section 4, whether the VCSEL operates with type I or type II LFF is mainly dependent on the linear cavity anisotropies.

A second difference between the model of Ref. 21 and Eqs. (1)-(4) consists in the inclusion of a self-gainsaturation coefficient in the model of Ref. 21. Our numerical study indicates that the gain-saturation coefficient increases the damping of the fast pulsating dynamics that underlie the slow LFF time traces (see Section 5), but is not responsible for the determination of the type of LFF dynamics.

Figures 1(d) and 2(d) show the degree of polarization $F P^{26}$ as a function of time after averaging on $1 \mathrm{~ns}$ for type
I or type II LFF, respectively. $F P$ is defined on the basis of the Stokes parameters $s_{0}$ to $s_{3}$, which are computed from

$$
\begin{aligned}
& s_{0}=\left|E_{x}\right|^{2}+\left|E_{y}\right|^{2}, \\
& s_{1}=2 \operatorname{Re}\left\{E_{x}^{*} E_{y}\right\}, \\
& s_{2}=2 \operatorname{Im}\left\{E_{x}^{*} E_{y}\right\}, \\
& s_{3}=\left|E_{x}\right|^{2}-\left|E_{y}\right|^{2} .
\end{aligned}
$$

$F P$ is then given by

$$
F P=\frac{\left\langle s_{1}\right\rangle^{2}+\left\langle s_{2}\right\rangle^{2}+\left\langle s_{3}\right\rangle^{2}}{\left\langle s_{0}\right\rangle^{2}},
$$

in which the Stokes parameters are averaged over a definite time span. In Figs. 1 and 2, averaging is done on 1 ns, as mentioned above. FP ranges between 0 for polarization-incoherent light and 1 for polarizationcoherent light. The two LFF types may be distinguished by the behavior of $F P$. In type II LFF, $F P$ is close to 1 in the time span between two drops of the dominant mode. Analysis of the Stokes parameters confirms that the light is close to linearly polarized along $y$. Once the power drop occurs FP decreases strongly as a result of timedependent fluctuations of the light polarization, then recovers again to 1 before the next power drop. By contrast, in type I LFF, FP is always close to zero: The light polarization fluctuates strongly with time.

\section{EFFECT OF BIREFRINGENCE AND GAIN ANISOTROPY: TRANSITION FROM ONE TYPE OF LFF TO THE OTHER}

The type I LFF reported in Ref. 20 occurs for internal parameters such that both polarization modes of the solitary VCSEL are unstable (which is favored by low $\gamma_{s}{ }^{27}$ ). We find that type I LFF may occur also in parameter regions where both modes are stable (which is favored by large $\gamma_{s}$ ) or where only one polarization mode is stable. In this last case optical feedback may turn on the unstable polarization mode, leading to either type I or type II LFF. Which of the two LFF types occurs depends on $\gamma_{a}$ and $\gamma_{p}$. The transition between both types of LFF is illustrated in Fig. 3. Figure 3 shows time traces of the total intensity $I_{\text {tot }}$, the $x$-LP mode intensity $I_{x}$ and the $y$-LP mode intensity $I_{y}$, after averaging on $1 \mathrm{~ns}$, for different values of $\gamma_{a}$ and $\gamma_{p}$ while the other laser and feedback parameters remain fixed. Since the total averaged powers of Figs. 1 and 2 are significantly different, one might suspect that type II LFF can be observed around the threshold for one polarization at low injection, whereas type I LFF appears at moderate injection current with symmetric oscillation conditions for the two polarization modes. However our numerical simulations in Fig. 3 show that both types of LFF might occur for the same injection current $\mu$ and adequate values of $\gamma_{a}$ and $\gamma_{p}$.

Type II LFF is favored by dichroism and birefringence. $\gamma_{a}$ models the different gain-to-loss ratio of the $x$ - and $y$-polarized modes $\left(\gamma_{a}>0\right.$ gives the $y$-mode a lower threshold). For parameters such that both modes compete with nearly equal mean power (type I LFF), increas- 

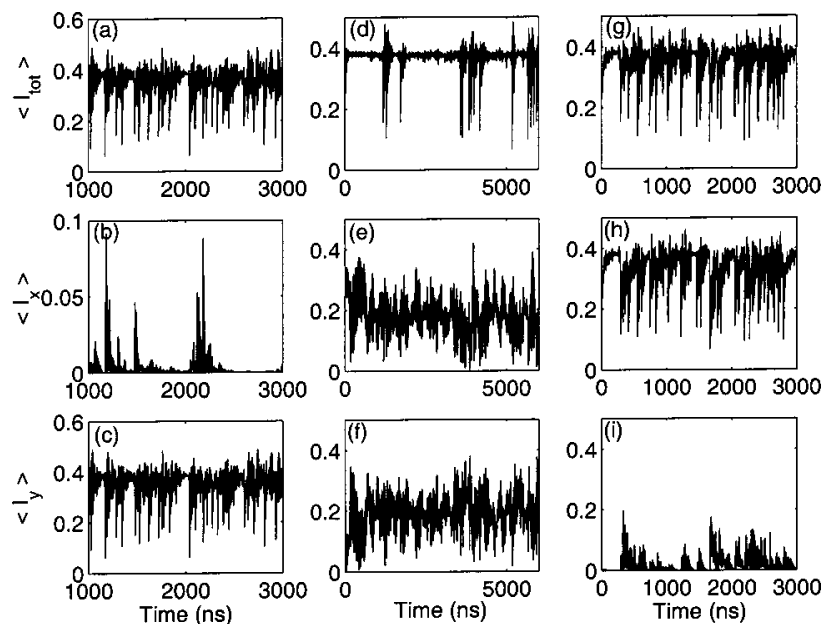

Fig. 3. Transitions from type I to type II LFF, and vice versa, for different values of $\gamma_{a}$ and $\gamma_{p}$. The other laser parameters remain fixed with the following values: $\mu=1.2, f=40 \mathrm{GHz}, \gamma_{s}$ $=50 \mathrm{~ns}^{-1}, \tau=3 \mathrm{~ns}, \beta_{\mathrm{sp}}=10^{-6} \mathrm{~ns}^{-1}$. (a), (d), and (g) show the time trace of the total intensity $I_{\text {tot }}$ after averaging on $1 \mathrm{~ns}$. (b), (e), and (h) show the averaged $x$-LP mode intensity $I_{x}$. (c), (f) and (i) show the averaged $y$-LP mode intensity $I_{y}$. (a)-(c) correspond to type II LFF with $\gamma_{a}=1 \mathrm{~ns}^{-1}, \gamma_{p}=0.1 \mathrm{~ns}^{-1}$. (d)-(f) correspond to type I LFF with $\gamma_{a}=0.1 \mathrm{~ns}^{-1}, \gamma_{p}=0.1 \mathrm{~ns}^{-1}$. (g)-(i) correspond to type II LFF with $\gamma_{a}=0.1 \mathrm{~ns}^{-1}, \quad \gamma_{p}$ $=15 \mathrm{~ns}^{-1}$.

ing $\gamma_{a}$ may cause the $y$-mode to be dominant and the $\mathrm{x}$-mode to be depressed, i.e., a transition to type II LFF. This transition from type I LFF to type II LFF is shown in Figs. 3(a)-(f). Figures 3(d)-(f) correspond to type I LFF but increasing the dichroism leads to type II LFF [Figs. $3(\mathrm{a})-(\mathrm{c})]$ in which the $x$-LP mode is depressed.

Varying the birefringence parameter $\gamma_{p}$ might also lead to a transition from one type of LFF to the other. When $\gamma_{p}>0$ the $x$-mode has the lower frequency and may be favored because of the effect of the saturable dispersion associated with the $\alpha$-factor. ${ }^{27}$ With feedback, for parameters such that both modes compete with equal power, increasing $\gamma_{p}$ leads to a transition to type II LFF in which the $x$-mode is the dominant mode and the $y$-mode is the depressed one. Such a transition from type I LFF to type II LFF is shown in Figs. 3(d)-(i). Figures 3(d)-(f) correspond to type I LFF but increasing the birefringence leads to type II LFF [Figs. 3(g)-(i)] in which the $y$-LP mode is depressed.

It is important to point out that for large $\gamma_{a}$ or large $\gamma_{p}$, to observe bursts of power in the depressed mode, spontaneous-emission-noise terms have to be included in Eq. (1). In the absence of noise, for large enough $\gamma_{a}$ or $\gamma_{p}$, the depressed mode after a transient turns off and remains off. Stochastic processes in the rate equations are therefore a minimal requirement to observe type II LFF. Figure 3 suggests also that either varying $\gamma_{a}$ or $\gamma_{p}$ would be sufficient to observe a transition from type I to type II LFF, i.e., that both linear anisotropies are not needed to observe the two types of LFF. We have indeed verified numerically that keeping $\gamma_{a}$ equal to zero and varying $\gamma_{p}$ might yield either type I LFF (at low birefringence) or type II LFF (at large birefringence). Furthermore if we keep $\gamma_{p}$ equal to zero and vary $\gamma_{a}$ this might yield either type I LFF (at low dichroism) or type II LFF (at large dichroism).

An experimental confirmation of the influence of the linear cavity anisotropies on the type of LFF dynamics deserves techniques to induce and control birefringence and dichroism of the VCSEL cavity. At least two techniques may be applied in that sense. First the so-called hot spot technique makes use of an external collimated beam and induces birefringence through elasto-optic effects. ${ }^{28}$ Second Panajotov et al. reported control of VCSEL polarization and cavity anisotropies through application of strain on the VCSEL mounting. ${ }^{29}$

\section{FAST POLARIZATION DYNAMICS}

Next we analyze the behavior of the fast-pulsing modal and total intensities during a power drop. As indicated in Ref. 17 the LFF regime is the time-averaged envelope of a train of pulses. An interesting question that deserves investigation is the analysis of the correlation between the intensity pulses emitted by both LP modes. Figures 4(a)-(b) display the results for type I LFF. Figure 4(a) shows the modal intensities $I_{x}$ (black) and $I_{y}$ (gray) when a total (and modal) power drop occurs. It shows that the modal intensities exhibit an antiphase dynamic before the power drop but after the drop occurs they synchronize and emit pulses in phase departing from the noise level. However we observe that when the $x$-LP mode intensity $I_{x}$ increases, the $y$-LP mode intensity $I_{y}$ decreases, and vice versa. The $x$ - and $y$-LP modes exchange energy on a slow ns time scale, which corresponds to a partial antiphase behavior. Before the power drop (after the recovery process of the total power), the total intensity will be nearly constant since the modes are in antiphase. But when a drop occurs the total intensity will
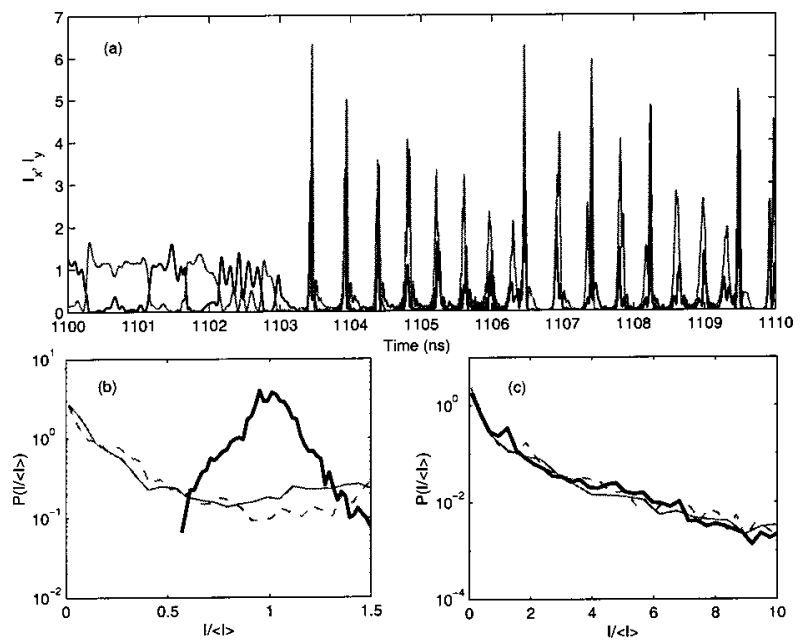

Fig. 4. Fast-pulsating dynamics of total and modal intensities for type I LFF. (a) shows the unaveraged $I_{x}$ (black curve) and $I_{y}$ (gray curve), and corresponds to an enlargement of the LFF time trace of Fig. 1. Both modes are in partial antiphase before the power drop while exhibiting in-phase pulsating output just after the drop, hence during the recovery process. (b) and (c) show the PDFs of the total (heavy black curve) and modal ( $x$, thin gray curve; $y$, dashed gray curve) intensities for a time span of $30 \mathrm{~ns}$ before (b) and after (c) the power drop. 

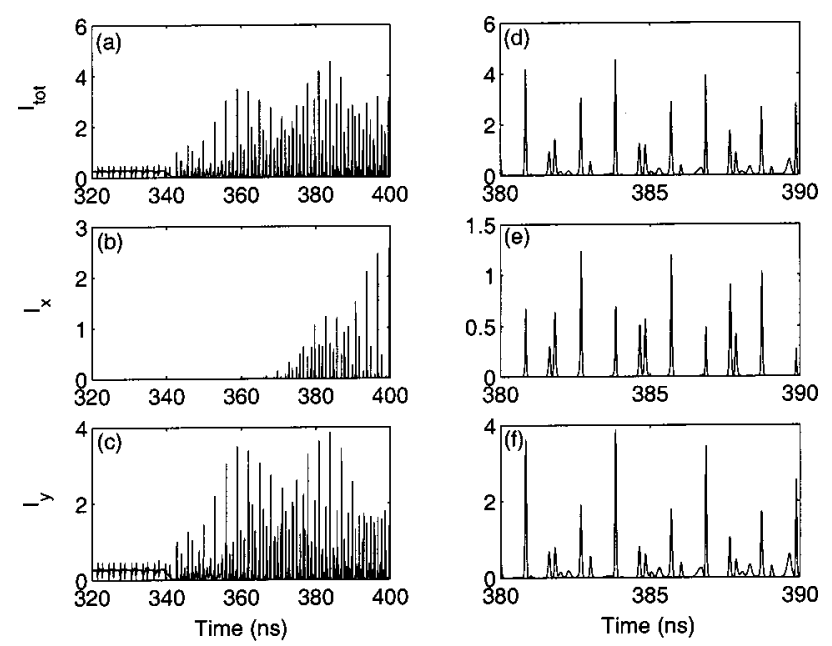

Fig. 5. Fast-pulsating dynamics of (a), (d) $I_{\text {tot }}$, (b), (e) $I_{x}$, and (c), (f) $I_{y}$ for type II LFF. The unaveraged intensities are plotted as a function of time in the time interval corresponding to one power drop of Fig. 2. (d)-(f) are enlargements of (a)-(c), respectively, to clarify the in-phase pulsating dynamics of the LP modes after the power drop.

display a train of pulses departing from the noise level similar to what occurs in the modal intensities, since modes emit in phase.

We gain insight into the correlation properties of the LP-mode intensities in the LFF regime by computing, as shown in Figs. 4(b) and (c), the probability distribution functions (PDFs) of the total (heavy black curve), $x$-LP mode intensity (thin gray curve), and $y$-LP mode intensity (dashed gray curve) for a 30-ns time interval before (b) and after (c) the power drop. For clarity the intensities $I$ are normalized by their corresponding mean values $\langle I\rangle$. In both cases (b) and (c) the PDFs of the modal intensities monotonically decay from the noise level, indicating emission of pulses with the most probable intensity close to the noise level. Before a power drop, the PDF of the total intensity displays a peak around its mean intensity value. In that case the total intensity exhibits small fluctuations around its mean value, since LP modes are in partial antiphase before a power drop. In contrast the PDF of the total intensity monotonically decays for a time interval just after the power drop, i.e., during the power recovery process, since the two LP modes emit pulses in phase and the corresponding total intensity exhibits pulses in phase with the LP modes.

Coexistence of in-phase and partial-antiphase dynamics is reported here for the first time in a polarizationunstable semiconductor laser. This topic has attracted much interest in connection with delayed laser systems because of its potential to contribute to the understanding of the influence of a multimode behavior on the total intensity dynamics. Our results suggest that a combination of two types of phase coherence in the modal intensities is a general feature of delayed-multimode laser systems, including systems with two polarization modes, ${ }^{12,13}$ with multiple transverse modes, ${ }^{30}$ or EELs with many longitudinal modes, ${ }^{31,32}$ even if the physics underlying the excitation of modes is different.

Figures 5(a)-(f) display the results for type II LFF. Before the drop the depressed polarization is off, and it rises with an in-phase pulsing behavior after the drop of the dominant mode. The partial synchronization of pulses in the polarized modes after a power drop is therefore a common feature for both types of LFFs. In Figs. 5(a)-(f) the power burst in the depressed mode is delayed with respect to the power drop in the dominant mode. However we have also observed in the same time trace of LFF bursts of the depressed mode which are simultaneous with drops of the dominant mode. Our results suggest that the delay between the drop in the dominant LP mode and the burst of the other depressed LP mode is a random variable with a nonzero mean value. The statistical properties of that variable delay is a subject for future work. Since spontaneous-emission noise plays an important role in the dynamics of the depressed mode, we speculate that the bursts are due to the complex interplay of noise and carrier dynamics: The increase in the carrier population that follows the drop of the dominant mode benefits the depressed mode which then rises with a burst from the noise level.

In agreement with Ref. 20 we find numerically that spontaneous-emission noise is not needed to observe type I LFF, in contrast with type II LFF. We therefore propose a first deterministic explanation for type I LFF. We analyze the route to LFF by computing bifurcation diagrams of the $x$ - and $y$-linearly polarized intensities as a function of the feedback rate $f$; see Fig. 6 . Figure 7 shows typical dynamics for different values of the feedback rate and illustrates the bifurcation study of Fig. 6. Initially for small feedback rates in Fig. 6, the route to LFF involves emission of both $x$ - and $y$-polarized modes. We calculate the optical spectrum of the $x$ - and $y$-polarized fields, finding that they have the same optical frequency, i.e., it is an elliptically polarized steady state; see Fig. 7(a). For increasing feedback Fig. 6 shows a bifurcation cascade alternating between elliptically polarized steady states and regions of time-periodic [see Fig. 7(b)] or even chaotic dynamics [see Fig. 7(c)]. At still larger feedback rates, windows of chaos broaden and lead to type I LFF, as shown in Figs. 7(d)-(f). This route to LFF in VCSELs
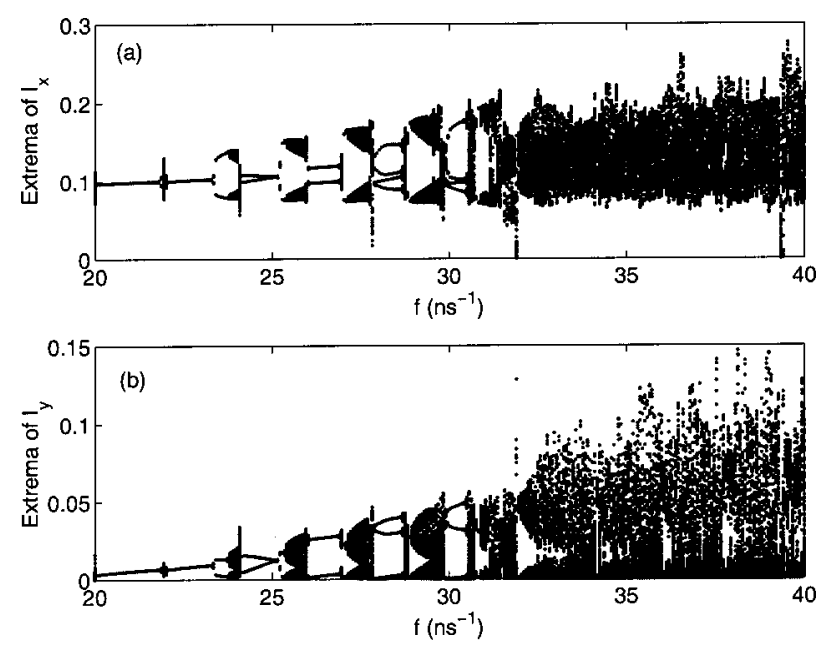

Fig. 6. Bifurcation diagram of (a) $I_{x}$ and (b) $I_{y}$ as a function of the feedback rate $f$ showing one possible route to type I LFF. The parameters are $\gamma_{a}=0.5 \mathrm{~ns}^{-1}, \gamma_{p}=8 \mathrm{~ns}^{-1}, \gamma_{s}=10 \mathrm{~ns}^{-1}$, $\mu=1.03, \beta_{\mathrm{sp}}=0, \tau=1 \mathrm{~ns}$. 

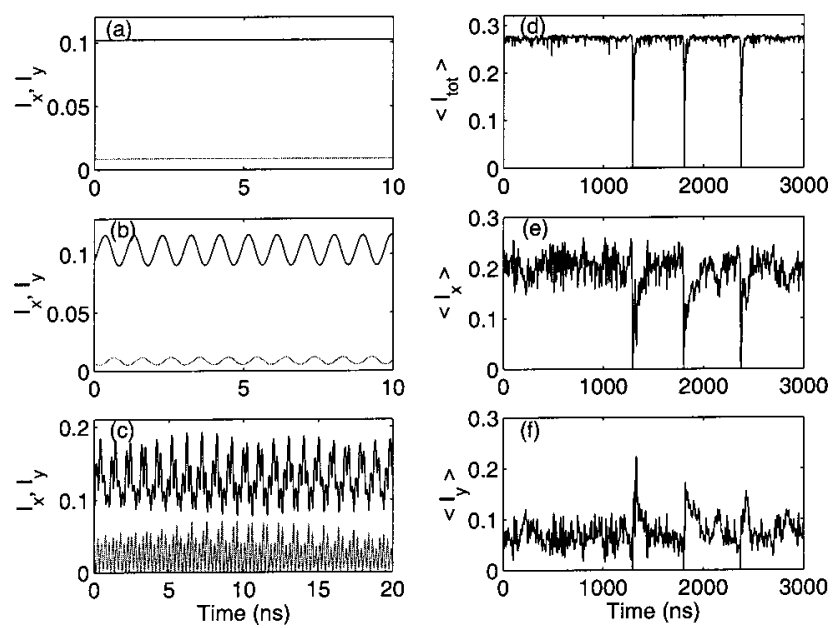

Fig. 7. Time traces of $I_{x}$ (black curves) and $I_{y}$ (gray curves) for (a) $f=23 \mathrm{~ns}^{-1}$, (b) $f=23.2 \mathrm{~ns}^{-1}$, (c) $f=34 \mathrm{~ns}^{-1}$. (d)-(f) show $I_{\text {tot }}, I_{x}$, and $I_{y}$, respectively, after averaging over $1 \mathrm{~ns}$ for $f$ $=60 \mathrm{~ns}^{-1}$. The figure shows type I LFF occurring after the bifurcation cascade of Fig. 6 . The other parameters are as in Fig. 6.

differs from that observed in conventional polarizationstable EELs. ${ }^{33}$ As previously reported ${ }^{20}$ the steady states of the delayed VCSEL system correspond to external-cavity modes that are either linearly polarized along $x$ or $y$ or elliptically polarized. Our results indicate that elliptically polarized external-cavity modes appear in the bifurcation route to LFF, and therefore motivate further theoretical studies on linear-stability analysis of external-cavity modes in the delayed-VCSEL problem.

\section{ALTERNATIVE MODEL}

Several different models have been used to study polarization dynamics in single-transverse-mode VCSELs. A typical alternative approach to the SFM model for solitary VCSELs is to consider a two-mode-rate-equations model including self- and cross-saturation coefficients. ${ }^{19}$ The gain-saturation coefficients model in a phenomenological way different coupling processes between polarization modes such as those induced by spatial hole burning that is the result of carrier diffusion. The polarization stability is determined by the values of the self- and crosssaturation coefficients. Such a model can be extended to optical feedback by use of the Lang-Kobayashi approach yielding a delayed-feedback term in the field equations as in Eqs. (1) and (2). The model has been successfully used to describe for example polarization self-modulation in VCSELs coupled to a polarization-rotating optical feedback ${ }^{10,12-14}$ and to study optical-feedback-induced polarization switchings. ${ }^{7,34}$

The model equations are written as

$$
\begin{aligned}
\dot{E}_{x}= & \kappa(1-i \alpha)\left(N F_{x}-1\right) E_{x} \\
& -\left(\gamma_{a}+i \gamma_{p}\right) E_{x}+f F_{x}(t-\tau) \exp \left(-i \phi_{f}\right) \\
& +\left(\beta_{\mathrm{sp}} N / 2\right)^{1 / 2} \xi_{1}+\left(\beta_{\mathrm{sp}} N / 2\right)^{1 / 2} \xi_{2},
\end{aligned}
$$

$$
\begin{aligned}
\dot{E}_{y}= & \kappa(1+i \alpha)\left(N F_{y}-1\right) E_{y} \\
& +\left(\gamma_{a}+i \gamma_{p}\right) E_{y}+f E_{y}(t-\tau) \exp \left(-i \phi_{f}\right) \\
& -i\left(\beta_{\mathrm{sp}} N / 2\right)^{1 / 2} \xi_{1}+i\left(\beta_{\mathrm{sp}} N / 2\right)^{1 / 2} \xi_{2}, \\
\dot{N}= & -\gamma_{N}\left[N-\mu+N\left(\left|E_{x}\right|^{2}+\left|E_{y}\right|^{2}\right)\right],
\end{aligned}
$$

with

$$
F_{x, y}=1-\epsilon_{x x, y x}\left|E_{x}\right|^{2}-\epsilon_{x y, y y}\left|E_{y}\right|^{2} .
$$

$\epsilon_{x x}, \epsilon_{y y}$ are self-gain-saturation coefficients and $\epsilon_{x y}, \epsilon_{y x}$ are cross-gain-saturation coefficients. Linear cavity anisotropies $\gamma_{a}$ and $\gamma_{p}$ are included in the model since Section 4 has shown they were important in observing both types of LFF. Except for the inclusion of $\gamma_{a}$ and $\gamma_{p}$, Eqs. (10)-(13) are identical to those of the model used in Refs. 12-14 if one considers the different normalization of the field and the carrier variable. ${ }^{27}$

Masoller and Abraham have analyzed such a two-mode model in the framework of the LFF regime. ${ }^{20}$ They have found that type I LFF can be observed provided that nonzero gain-saturation coefficients are included in the rate equations. As can be expected from our previous analysis, we now show that such a model could also reproduce type II LFF depending on the values of the linear cavity anisotropies. First our numerical simulations indicate that to observe LFF, nonzero values of the cross-gainsaturation coefficients should be included in Eqs. (10)(13), but the self-gain saturation coefficients may be omitted. Our results therefore substantiate previous numerical studies on that delayed-VCSEL mode ${ }^{20}$ by discriminating between the influence of the cross-gain saturation coefficient and that of the self-gain saturation coefficient on inducing LFF.

Figure 8 illustrates type II LFF obtained with the model of Eqs. (10)-(13) for the same laser and feedback parameters as in Fig. 2 and with $\epsilon_{x x}=\epsilon_{y y}=0$ and $\epsilon_{x y}$ $=\epsilon_{y x}=0.01$. It shows that the $y$-LP mode intensity exhibits power drops similar to the dynamics of the total intensity, and that the $x$-LP mode is strongly depressed but
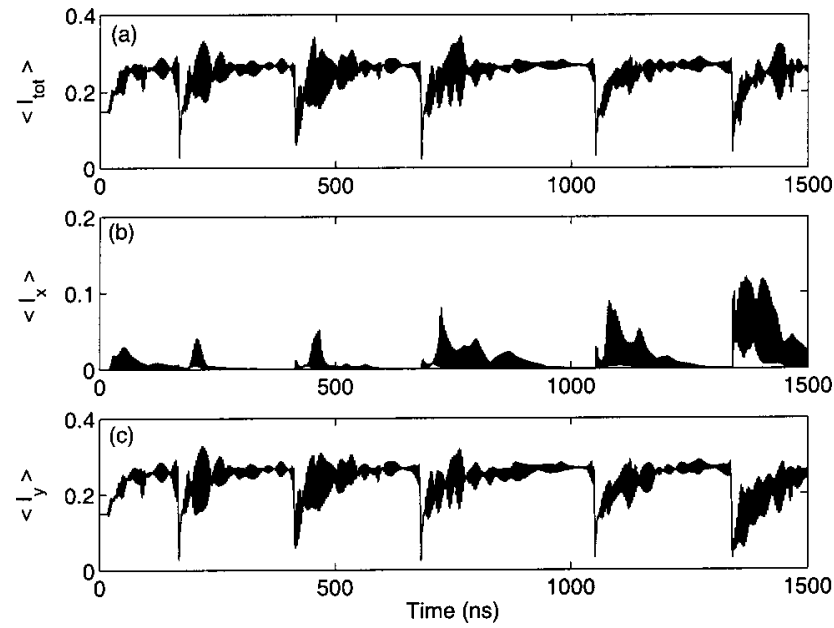

Fig. 8. Time traces of (a) the total intensity $I_{\text {tot }}$ and (b), (c) the modal intensities $I_{x}$ and $I_{y}$, respectively, after averaging on $1 \mathrm{ns,}$ showing type II LFF with the model of rate Eqs. (10)-(13). The laser and feedback parameters are the same as in Fig. 1 with $\epsilon_{x x}=\epsilon_{y y}=0$ and $\epsilon_{x y}=\epsilon_{y x}=0.01$. 
exhibits power bursts consequent to the drops in the main mode. Furthermore we arrive at the same conclusion concerning the influence of the linear anisotropies as that of Section 4: Type II LFF is favored by large $\gamma_{a}$ and/or large $\gamma_{p}$. Our results, combined with those of Ref. 20, show that both types of LFF dynamics are found with the rate Eqs. (10)-(13). Our conclusions also match those of Section 4 concerning the influence of the linear cavity anisotropies on the type of LFF dynamics.

\section{CONCLUSION}

In summary numerical solutions of a model for VCSELs with optical feedback show that VCSELs might exhibit two qualitatively different regimes of LFFs that can be clearly distinguished by the polarization behavior. We have shown that the degree of polarization FP allows a clear discrimination between the two types of LFF dynamics. That approach leads to a new insight not suggested by the previous approach of Refs. 20, 21 that could be tested experimentally. The two types of LFF dynamics exist in a wide range of parameters and for two different physical models. Furthermore we have shown that a transition from one type of LFF to the other might be observed by modifying the frequency splitting and/or the gain-to-loss ratio of the modes. Since only type II LFF has been experimentally reported in VCSELs, our results motivate new experiments on VCSELs coupled to long external cavities. In light of our results special attention may be given to the influence of the linear cavity anisotropies. Our results also analyze an example of vectorial chaos in lasers which might be of use for chaotic communication systems.

\section{ACKNOWLEDGMENTS}

M. Sciamanna is a Research Fellow of the Fonds National de la Recherche Scientifique (FNRS, Belgium). Our research is also supported by the Interuniversity Attraction Pole (IAP) program of the Belgian government. C. Masoller is partially supported by PEDECIBA (Proyecto de Desarrollo de Ciencias Basicas, Uruguay) and CSIC (Comision Sectorial de Investigacion Cientifica, Uruguay).

M. Siamanna may be reached by e-mail at Sciamanna @telecom.fpms.ac.be.

\section{REFERENCES}

1. H. Soda, K. Iga, C. Kitahara, and Y. Suematsu, "GaInAsP/ InP surface emitting injection lasers," Jpn. J. Appl. Phys. 18, 2329-2330 (1979).

2. K. Iga, "Surface-emitting laser-Its birth and generation of new optoelectronics field," IEEE J. Sel. Top. Quantum Electron. 1, 667-673 (1995).

3. C. J. Chang-Hasnain, J. P. Harbison, G. Hasnain, A. C. Von Lehmen, L. T. Florez, and N. G. Stoffel, "Dynamic, polarization, and transverse mode characteristics of vertical-cavity surface-emitting lasers," IEEE J. Quantum Electron. 24, 1402-1409 (1991).

4. H. Kawaguchi, Bistabilities and Nonlinearities in Laser Diodes (Artech House, Norwood, Mass., 1994).
5. Y. C. Chung and Y. H. Lee, "Spectral characteristics of vertical-cavity surface-emitting lasers with external optical feedback," IEEE Photonics Technol. Lett. 3, 597-599 (1991).

6. P. Besnard, F. Robert, M. L. Chares, and G. M. Stephan, "Theoretical modeling of vertical-cavity surface-emitting lasers with polarized optical feedback," Phys. Rev. A 56, 3191-3205 (1997).

7. L. Plouzennec, "Polarization gain dependence of verticalcavity surface-emitting lasers: phenomenological modeling and experiments," J. Appl. Phys. 87, 2649-2651 (2000).

8. S. Jiang, Z. Pan, M. Dagenais, R. A. Morgan, and K. Kojima, "High frequency polarization self-modulation in vertical-cavity surface-emitting lasers," Appl. Phys. Lett. 63, 3545-3547 (1993).

9. F. Robert, P. Besnard, M. L. Chares, and G. Stephan, "Polarization modulation dynamics of vertical-cavity surfaceemitting lasers with an extended cavity,” IEEE J. Quantum Electron. 33, 2231-2239 (1997).

10. H. Li, A. Hohl, A. Gavrielides, H. Hou, and K. D. Choquette, "Stable polarization self-modulation in vertical-cavity surface-emitting lasers," Appl. Phys. Lett. 72, 2355-2357 (1998).

11. C. Masoller and N. B. Abraham, "Polarization dynamics of vertical-cavity surface-emitting lasers with optical feedback through a quarter-wave plate," Appl. Phys. Lett. 74, 10781080 (1999).

12. M. Sciamanna, F. Rogister, O. Deparis, P. Mégret, M. Blondel, and T. Erneux, "Bifurcation to polarization selfmodulation in vertical-cavity surface-emitting lasers," Opt. Lett. 27, 261-263 (2002).

13. M. Sciamanna, F. Rogister, O. Deparis, P. Mégret, M. Blondel, and T. Erneux, "Bifurcation to polarization selfmodulation in vertical-cavity surface-emitting lasers: errata," Opt. Lett. 27, 875 (2002).

14. M. Sciamanna, T. Erneux, F. Rogister, O. Deparis, P. Mégret, and M. Blondel, "Bifurcation bridges between external-cavity modes lead to polarization self-modulation in vertical-cavity surface-emitting lasers," Phys. Rev. A 65, 041801/1(R)-041801/4(R) (2002).

15. M. Sciamanna, K. Panajotov, P. Mégret, H. Thienpont, M. Blondel, and I. Veretennicoff, "Optical feedback induced polarization mode-hopping in vertical-cavity surface-emitting lasers," presented at the OSA Annual Meeting, Orlando, Florida (October 3, 2002).

16. G. D. Van Wiggeren and R. Roy, "Communicating with dynamically fluctuating states of light polarization," Phys. Rev. Lett. 88, 097903/1-097903/4 (2002).

17. I. Fischer, T. Heil, and W. Elsässer, "Emission dynamics of semiconductor lasers subject to delayed optical feedback: an experimentalist's perspective," in Fundamental Issues of Nonlinear Dynamics, B. Krauskopf and D. Lenstra, eds. (AIP Conference Proceedings, Melville, New York, 2000), Vol. 548, pp. 218-237.

18. M. San Miguel, Q. Feng, and J. V. Moloney, "Light polarization dynamics in surface-emitting semiconductor lasers," Phys. Rev. A 52, 1728-1739 (1995).

19. J. Danckaert, B. Nagler, J. Albert, K. Panajotov, I. Veretennicoff, and T. Erneux, "Minimal rate equations describing polarization switching in vertical-cavity surface-emitting lasers," Opt. Comm. 201, 129-137 (2002).

20. C. Masoller and N. B. Abraham, "Low-frequency fluctuations in vertical-cavity surface-emitting semiconductor lasers with optical feedback," Phys. Rev. A 59, 3021-3031 (1999).

21. M. Giudici, S. Balle, T. Ackemann, S. Barland, and J. R. Tredicce, "Polarization dynamics of vertical-cavity surfaceemitting lasers with optical feedback: experiment and model," J. Opt. Soc. Am. B 16, 2114-2123 (1999).

22. J. Y. Law and G. P. Agrawal, "Effects of optical feedback on static and dynamic characteristics of vertical-cavity surface-emitting lasers," IEEE J. Sel. Top. Quantum Electron. 3, 353-358 (1997).

23. P. Spencer, C. R. Mirasso, and K. A. Shore, "Effect of strong optical feedback on vertical-cavity surface-emitting lasers," IEEE Photonics Technol. Lett. 10, 191-193 (1998). 
24. J. Dellunde, A. Valle, L. Pesquera, and K. A. Shore, "Transverse-mode selection and noise properties of external-cavity vertical-cavity surface-emitting lasers including multiple-reflection effects,” J. Opt. Soc. Am. B 16, 2131-2139 (1999).

25. R. Lang and K. Kobayashi, "External optical feedback effects on semiconductor injection laser properties," IEEE J. Quantum Electron. 16, 347-355 (1980).

26. R. M. A. Azzam and N. M. Bashara, Ellipsometry and Polarized Light, (North-Holland, Amsterdam, 1977).

27. J. Martin-Regalado, F. Prati, M. San Miguel, and N. B. Abraham, "Polarization properties of vertical-cavity surface-emitting lasers," IEEE J. Quantum Electron. 33, 765-783 (1997).

28. A. K. Jansen van Doorn, M. P. van Exter, and J. P. Woerdman, "Tailoring the birefringence in a vertical-cavity surface-emitting laser," Appl. Phys. Lett. 69, 3635-3637 (1996).

29. K. Panajotov, B. Nagler, G. Verschaffelt, A. Georgievski, H. Thienpont, J. Danckaert, and I. Veretennicoff, "Impact of in-plane anisotropic strain on the polarization behavior of vertical-cavity surface-emitting lasers,” Appl. Phys. Lett. 77, 1590-1592 (2000)

30. M. S. Torre, C. Masoller, and P. Mandel,“Transverse mode dynamics in vertical-cavity surface-emitting lasers with optical feedback," Phys. Rev. A (to be published).

31. E. A. Vikotorov and P. Mandel, "Low frequency fluctuations in a multimode semiconductor laser with optical feedback," Phys. Rev. Lett. 85, 3157-3160 (2000).

32. F. Rogister, P. Mégret, O. Deparis, and M. Blondel, "Coexistence of in-phase and out-of-phase dynamics in a multimode external-cavity laser diode operating in the lowfrequency fluctuations regime," Phys. Rev. A 62, 061803/ $1(\mathrm{R})-061803 / 4(\mathrm{R})(2000)$

33. A. Hohl and A. Gavrielides, "Bifurcation cascade in a semiconductor laser subject to optical feedback," Phys. Rev. Lett. 82, 1148-1151 (1999).

34. P. Besnard, M. Chares, G. Stephan, and F. Robert, "Switching between polarization modes of a vertical-cavity surfaceemitting laser by isotropic optical feedback," J. Opt. Soc. Am. B 16, 1059-1063 (1999). 